\title{
Evolution equation for entanglement of assistance
}

\author{
Zong-Guo Li, ${ }^{1}$ Ming-Jing Zhao, ${ }^{2}$ Shao-Ming Fei, ${ }^{2}$ and W. M. Liu ${ }^{1}$ \\ ${ }^{1}$ Beijing National Laboratory for Condensed Matter Physics, \\ Institute of Physics, Chinese Academy of Sciences, Beijing 100190, China \\ ${ }^{2}$ School of Mathematical Sciences, Capital Normal University, Beijing 100048, China
}

\begin{abstract}
We investigate the time evolution of the entanglement of assistance when one subsystem undergoes the action of local noisy channels. A general factorization law is presented for the evolution equation of entanglement of assistance. Our results demonstrate that the dynamics of the entanglement of assistance is determined by the action of a noisy channel on the pure maximally entangled state, in which the entanglement reduction turns out to be universal for all quantum states entering the channel. This single quantity will make it easy to characterize the entanglement dynamics of entanglement of assistance under unknown channels in the experimental process of producing entangled states by assisted entanglement.
\end{abstract}

PACS numbers: 03.67.Mn, 03.65.Ud, 03.67.Bg, 03.65.Ta

\section{INTRODUCTION}

In quantum information, entanglement is a vital resource for quantum cryptography and quantum computation [1, 2]. More specially the distribution of bipartite entanglement is a key ingredient for performing certain quantum-information processing tasks such as teleportation. Therefore, the creation and distribution of entanglement is of central interest in quantum information processing.

There is an alternative to producing bipartite entanglement by reducing a multipartite entangled state to an entangled state with fewer parties (e.g. bipartite) via measurements on the other parties. Such producing of entanglement, called "assisted entanglement", is a special case of the localizable entanglement [3], which is especially important for quantum communication, where quantum repeaters are needed to establish bipartite entanglement over a long length scale [4]. The entanglement in this produced entangled state is quantified by the entanglement of assistance (EOA) which is defined in Refs. [5, [6].

However, in the transition and store of quantum entangled state, we inevitably encounter some interactions of the multipartite quantum state under consideration with its environment. For example, in an experimental preparation of entanglement for a quantum information processing task, suppose a supplier of entanglement, named "Charlie", creates entanglement between two distant parties, Alice and Bob, by the assisted entanglement. Suppose also that Charlie starts with a pure tri-qudit entangled state such as the Greenberger-Horne-Zeilinger (GHZ) state 7] and sends, via quantum channels, two of the three qudits to Alice and Bob. Due to the environmental interaction, the quantum channels generally make the pure tripartite entangled state degrade to a mixed tripartite entangled state. These undesired couplings give rise to decoherence, which may decrease the entanglement in the created state when the particles propagate. Therefore, it is of great practical importance to investi- gate the dynamics of entanglement of assistance for the quantum system under the influence of decoherence.

Recently, the entanglement evolution has been studied extensively for bipartite system under the influence of local decoherence. In stead of deducing the evolution of entanglement from the time evolution of the state a direct relationship between the initial and final entanglement of a bipartite state has been explored in e.g., Refs. 8 10]. In this paper, we study the time evolution of EOA when one subsystem undergoes the action of an arbitrary noisy channel. Our results demonstrate that the dynamics of EOA is determined by the action of channel on the bipartite pure maximally entangled state. In particular, for a $2 \times 2 \times n_{3}$ pure quantum state with one qubit subsystem being subject to a quantum channel, we get a general factorization law for the evolution equation of EOA, i.e., the EOA of the final state can be expressed as the product of the initial EOA multiplied by the entanglement reduction of maximally entangled state after the process of the quantum channel's action. The latter quantity is universal for all initial states passing through this channel. We take generalized amplitude damping and phase damping channels as examples, and find that the sudden death of EOA exists in the evolution and is only determined by the entanglement evolution equation of the maximally entangled state entering this channel. Moreover, for the other cases with one subsystem undergoing the action of an arbitrary noisy channel, a similar relation is satisfied, with a sacrifice that the equality is replaced by an inequality.

This paper is organized as follows: We start by introducing a definition of EOA and further study the dynamics of EOA for $2 \times 2 \times n_{3}$ systems with a qubit being subject to a noisy channel. In Sec. III, the evolution equation of EOA is investigated for a general $d \times d \times n_{3}$ state with a local operation on the second subsystem. We apply the evolution equation to some cases and discuss the result in Sec. IV. Finally, in Sec. V, we conclude with a summary of our results. 


\section{EVOLUTION EQUATION OF EOA FOR $2 \times 2 \times n_{3}$ SYSTEMS}

A 2-qubit entangled state is the basic unit of quantum information. An important application of assisted entanglement is to create a 2-qubit entangled state from a $2 \times 2 \times n_{3}$ entangled state. Therefore, we firstly consider a pure $2 \times 2 \times n_{3}$ state shared by three parties referred to as Alice, Bob and Charlie. Charlie's aim is to maximize the entanglement of the state between Alice and Bob by performing a local operation on his system and communicating the result to Alice and Bob. For $2 \otimes 2$ systems, we take concurrence to quantify the entanglement and EOA then reduce to the concurrence of assistance [11, 12]. For a pure $2 \times 2 \times n_{3}$ state $|\psi\rangle_{A B C}$, the EOA is defined as follows:

$$
E_{a}\left(|\psi\rangle_{A B C}\right) \equiv E_{a}\left(\rho_{A B}\right) \equiv \max \sum_{i} p_{i} C\left(\left|\phi_{i}\right\rangle_{A B}\right),
$$

where $\rho_{A B}=\operatorname{Tr}_{C}\left(|\psi\rangle_{A B C}\langle\psi|\right)$, and $C\left(\left|\phi_{i}\right\rangle_{A B}\right)$ is the concurrence defined in Ref. [13]. The maximum runs over all possible pure-state decompositions of $\rho_{A B}=$ $\operatorname{Tr}_{C}\left[|\psi\rangle_{A B C}\langle\psi|\right]=\sum_{i} p_{i}\left|\phi_{i}\right\rangle_{A B}\left\langle\phi_{i}\right|$. Equation (11) quantifies the maximum average entanglement that Charlie can create between Alice and Bob because any pure-state decompositions of $\rho_{A B}$ can be realized by a generalized measurement performed by Charlie (for more details see [14, 15]). Thomas Laustsen et al. [11] have given an analytical expression for EOA,

$$
E_{a}\left(|\psi\rangle_{A B C}\right)=\operatorname{Tr} \sqrt{\sqrt{\rho_{A B}} \tilde{\rho}_{A B} \sqrt{\rho_{A B}}}=\sum_{i} \lambda_{i},
$$

where $\tilde{\rho}_{A B}=\sigma_{y} \otimes \sigma_{y} \rho_{A B}^{*} \sigma_{y} \otimes \sigma_{y}$ with $\sigma_{y}$ Pauli matrix, and $\lambda_{i}$ are the square roots of the eigenvalues of $\rho_{A B} \tilde{\rho}_{A B}$. In a similar way, for a mixed tripartite state $\rho_{A B C}$, the EOA is defined as the maximum achievable average concurrence shared by Alice and Bob after Charlie's assistance, depends on $\rho_{A B C}$ and thus not solely on $\rho_{A B}$.

Suppose $|\psi\rangle_{A B C}$ be the tripartite pure initial state, and the second subsystem undergo the action of a noisy channel. We will denote the noisy channel by $\mathcal{S}$ and take the positive-operator valued measure (POVM) measurements to describe the channel $\mathcal{S}$ thereafter. Then the final state of the system takes the form $\rho^{\prime}=(\mathbf{1} \otimes \mathcal{E} \otimes$ 1) $|\psi\rangle_{A B C}\langle\psi|$, which is usually mixed. In general, the EOA is difficult to solve for mixed states. For this state $\rho^{\prime}$ with one qubit having gone through a noisy channel, we have an analytical expression of EOA as shown in the following:

Theorem 1 For a pure $2 \times 2 \times n_{3}$ state $|\psi\rangle_{A B C}$, the second subsystem of which is undergoing the action of the channel $\mathcal{E}$, the EOC of the final state $\rho^{\prime}$ takes the form as the following equation:

$$
\begin{aligned}
& E_{a}\left[(\mathbf{1} \otimes \mathcal{E} \otimes \mathbf{1})|\psi\rangle_{A B C}\right] \\
= & E_{a}\left[|\psi\rangle_{A B C}\right] C\left[(\mathbf{1} \otimes \mathcal{E})\left|\phi^{+}\right\rangle\left\langle\phi^{+}\right|\right] \\
= & E_{a}\left(\rho_{A B}\right) C\left[(\mathbf{1} \otimes \mathcal{E})\left|\phi^{+}\right\rangle\left\langle\phi^{+}\right|\right],
\end{aligned}
$$

where $\left|\phi^{+}\right\rangle$is the bipartite maximally entangled state, $\left|\phi^{+}\right\rangle=1 / \sqrt{2}(|00\rangle+|11\rangle)$.

Proof: Suppose the channel $\mathcal{E}$ is denoted by the Kraus operators $\left\{K_{j}\right\}$ with $\sum_{j} K_{j}^{\dagger} K_{j}=\mathbf{1}$. For the tripartite pure state $|\psi\rangle_{A B C}$, there must be a set of optimal measurement $\left\{M_{i}\right\}$ for Charlie, such that the EOA $E_{a}\left[|\psi\rangle_{A B C}\langle\psi|\right]=\sum_{i} p_{i} C\left(\left|\psi_{i}\right\rangle\right)$ with the optimal pure-state decomposition $\left|\psi_{i}\right\rangle\langle\psi|=\operatorname{Tr}_{C}[(\mathbf{1} \otimes \mathbf{1} \otimes$ $\left.\left.\otimes M_{i}\right)|\psi\rangle_{A B C}\langle\psi|\left(\mathbf{1} \otimes \mathbf{1} \otimes M_{i}^{\dagger}\right)\right] / p_{i}$, and $p_{i}=\operatorname{Tr}[(\mathbf{1} \otimes$ $\left.\left.M_{i}\right)|\psi\rangle_{A B C}\langle\psi|\left(\mathbf{1} \otimes M_{i}^{\dagger}\right)\right]$. Considering the final state $\rho^{\prime}=\sum_{j}\left(\mathbf{1} \otimes K_{j} \otimes \mathbf{1}\right)|\psi\rangle_{A B C}\langle\psi|\left(\mathbf{1} \otimes K_{j}^{\dagger} \otimes \mathbf{1}\right)$, Charlie can also take the local measurement $\left\{M_{i}\right\}$ and correspondingly obtains the states shared by Alice and Bob, $\rho_{i}^{\prime}=\operatorname{Tr}_{C}\left[\sum_{j}\left(\mathbf{1} \otimes K_{j} \otimes M_{i}\right)|\psi\rangle_{A B C}\langle\psi|\left(\mathbf{1} \otimes K_{j}^{\dagger} \otimes M_{i}^{\dagger}\right)\right] / p_{i}^{\prime}=$ $\sum_{j}\left(\mathbf{1} \otimes K_{j}\right)\left|\psi_{i}\right\rangle\left\langle\psi_{i}\right|\left(\mathbf{1} \otimes K_{j}^{\dagger}\right) / p_{i}^{\prime}$, and $p_{i}^{\prime}=\operatorname{Tr}\left[\sum_{j}\left(\mathbf{1} \otimes K_{j} \otimes\right.\right.$ $\left.\left.M_{i}\right)|\psi\rangle_{A B C}\langle\psi|\left(\mathbf{1} \otimes K_{j}^{\dagger} \otimes M_{i}^{\dagger}\right)\right]=p_{i}$. Therefore, in virtue of the definition of EOA for mixed states, the EOA satisfies [16]

$$
\begin{aligned}
E_{a}\left(\rho^{\prime}\right) & \geq \sum_{i} p_{i} C\left[(\mathbf{1} \otimes \mathcal{E})\left|\psi_{i}\right\rangle\right] \\
& =\sum_{i} p_{i} C\left(\left|\psi_{i}\right\rangle\right) C\left[(\mathbf{1} \otimes \mathcal{E})\left|\phi^{+}\right\rangle\left\langle\phi^{+}\right|\right] \\
& =E_{a}\left(|\psi\rangle_{A B C}\right) C\left[(\mathbf{1} \otimes \mathcal{E})\left|\phi^{+}\right\rangle\left\langle\phi^{+}\right|\right],
\end{aligned}
$$

where $\left|\phi^{+}\right\rangle$is the 2-qubit maximally entangled state.

We next prove the reverse inequality also holds. We first assume that there is a set of optimal measurement $\left\{M_{i}^{\prime \prime}\right\}$ for Charlie such that

$$
E_{a}\left(\rho^{\prime}\right)=\sum_{i} p_{i}^{\prime \prime} C\left(\rho_{i}^{\prime \prime}\right)
$$

where $p_{i}^{\prime \prime}=\operatorname{Tr}\left[\sum_{j}\left(\mathbf{1} \otimes K_{j} \otimes M_{i}^{\prime \prime}\right)|\psi\rangle_{A B C}\langle\psi|\left(\mathbf{1} \otimes K_{j}^{\dagger} \otimes\right.\right.$ $\left.\left.M_{i}^{\prime \prime \dagger}\right)\right]$, and

$$
\begin{aligned}
& \rho_{i}^{\prime \prime} \\
= & \frac{\operatorname{Tr}_{C} \sum_{j}\left(\mathbf{1} \otimes K_{j} \otimes M_{i}^{\prime \prime}\right)|\psi\rangle_{A B C}\langle\psi|\left(\mathbf{1} \otimes K_{j}^{\dagger} \otimes M_{i}^{\prime \prime \dagger}\right)}{p_{i}^{\prime \prime}} \\
= & \sum_{j}\left(\mathbf{1} \otimes K_{j}\right) \tau_{i}^{\prime \prime}\left(\mathbf{1} \otimes K_{j}^{\dagger}\right) .
\end{aligned}
$$

Here $\tau_{i}^{\prime \prime}=\operatorname{Tr}_{C}\left(\left(\mathbf{1} \otimes \mathbf{1} \otimes M_{i}^{\prime \prime}\right)|\psi\rangle_{A B C}\langle\psi|\left(\mathbf{1} \otimes \mathbf{1} \otimes M_{i}^{\prime \prime \dagger}\right)\right) / p_{i}^{\prime \prime}$. In terms of the results in Ref. [8], the inequality $C((\mathbf{1} \otimes$ $\mathcal{E}) \rho) \leq C(\rho) C\left((\mathbf{1} \otimes \mathcal{E})\left|\phi^{+}\right\rangle\right)$holds for a 2-qubit mixed state $\rho$. We thus have

$$
\begin{aligned}
E_{a}\left(\rho^{\prime}\right) & =\sum_{i} p_{i}^{\prime \prime} C\left(\rho_{i}^{\prime \prime}\right) \\
& =\sum_{i} p_{i}^{\prime \prime} C\left((\mathbf{1} \otimes \mathcal{E}) \tau_{i}^{\prime \prime}\right) \\
& \leq C\left((\mathbf{1} \otimes \mathcal{E})\left|\phi^{+}\right\rangle\left\langle\phi^{+}\right|\right) \sum_{i} p_{i}^{\prime \prime} C\left(\tau_{i}^{\prime \prime}\right) .
\end{aligned}
$$


Due to $\rho_{A B}=\operatorname{Tr}_{C}\left(|\psi\rangle_{A B C}\langle\psi|\right)=\sum_{i} p_{i}^{\prime \prime} \tau_{i}^{\prime \prime}$ and the concavity of EOA for pure states, the following equation holds,

$$
E_{a}\left(|\psi\rangle_{A B C}\right) \geq \sum_{i} p_{i}^{\prime \prime} C\left(\tau_{i}^{\prime \prime}\right)
$$

Accordingly, we have

$$
\begin{aligned}
E_{a}\left(\rho^{\prime}\right) & \leq C\left((\mathbf{1} \otimes \mathcal{E})\left|\phi^{+}\right\rangle\left\langle\phi^{+}\right|\right) \sum_{i} p_{i}^{\prime \prime} C\left(\tau_{i}^{\prime \prime}\right) \\
& \leq E_{a}\left(|\psi\rangle_{A B C}\right) C\left((\mathbf{1} \otimes \mathcal{E})\left|\phi^{+}\right\rangle\left\langle\phi^{+}\right|\right) .
\end{aligned}
$$

Therefore, in terms of Eqs. (44) and (9), we obtain the equation,

$$
\begin{aligned}
& E_{a}\left[(\mathbf{1} \otimes \mathcal{E} \otimes \mathbf{1})|\psi\rangle_{A B C}\right] \\
= & E_{a}\left(|\psi\rangle_{A B C}\right) C\left[(\mathbf{1} \otimes \mathcal{E})\left|\phi^{+}\right\rangle\left\langle\phi^{+}\right|\right]
\end{aligned}
$$

The EOA reduction, with a one-sided noisy channel operating on Alice or Bob subsystem, is independent of the initial state $|\psi\rangle_{A B C}$ and completely determined by the channels action on the 2-qubit maximally entangled state. Thus, if we know the dynamics of the Bell states entanglement under a one-sided noisy channel, we then know the time evolution of EOA for any pure $2 \times 2 \times n_{3}$ initial states.

The result (3) can also be generalized for a mixed $2 \times$ $2 \times n_{3}$ initial state $\rho_{0}$.

Corollary 2 For a mixed $2 \times 2 \times n_{3}$ initial state $\rho_{0}$, with a one-sided noisy channel operating on Bob subsystem, we obtain its evolution equation of EOA,

$$
\begin{aligned}
& E_{a}\left((\mathbf{1} \otimes \mathcal{E} \otimes \mathbf{1}) \rho_{0}\right) \\
\leq & E_{a}\left(\rho_{0}\right) C\left[(\mathbf{1} \otimes \mathcal{E})\left|\phi^{+}\right\rangle\left\langle\phi^{+}\right|\right] .
\end{aligned}
$$

This corollary is proved as follows. Suppose there exists a set of optimal measurements for Charlie to create the state between Alice and Bob, $\left\{q_{i}, \tau_{i}\right\}$, such that the EOA for the state $\rho_{0}$ satisfies the equation $E_{a}\left(\rho_{0}\right)=$ $\sum_{i} q_{i} C\left(\tau_{i}\right)$. After the channel operates on the initial state, we assume that there is another set of optimal measurements for Charlie so that $E_{a}\left((\mathbf{1} \otimes \mathcal{E} \otimes \mathbf{1}) \rho_{0}\right)=$ $\sum_{i} q_{i}^{\prime} C\left((\mathbf{1} \otimes \mathcal{E}) \tau_{i}^{\prime}\right)$. Due to $\sum_{i} q_{i} \tau_{i}=\operatorname{Tr}_{C}\left(\rho_{0}\right)=\operatorname{Tr}_{C}[(\mathbf{1} \otimes$ $\left.\mathcal{E} \otimes \mathbf{1}) \rho_{0}\right]=\sum_{i} q_{i}^{\prime} \tau_{i}^{\prime}$, both $\left\{q_{i}, \tau_{i}\right\}$ and $\left\{q_{i}^{\prime}, \tau_{i}^{\prime}\right\}$ can be realized by the Charlie's measurements no matter whether the local quantum channel operates on the initial state. Therefore, we can prove $E_{a}\left((\mathbf{1} \otimes \mathcal{E} \otimes \mathbf{1}) \rho_{0}\right)=\sum_{i} q_{i}^{\prime} C((\mathbf{1} \otimes$ $\left.\mathcal{E}) \tau_{i}^{\prime}\right) \leq C\left[(\mathbf{1} \otimes \mathcal{E})\left|\phi^{+}\right\rangle\left\langle\phi^{+}\right|\right] \sum_{i} q_{i}^{\prime} C\left(\tau_{i}^{\prime}\right) \leq C[(\mathbf{1} \otimes$ $\left.\mathcal{E})\left|\phi^{+}\right\rangle\left\langle\phi^{+}\right|\right] \sum_{i} q_{i} C\left(\tau_{i}\right)=E_{a}\left(\rho_{0}\right) C\left[(\mathbf{1} \otimes \mathcal{E})\left|\phi^{+}\right\rangle\left\langle\phi^{+}\right|\right]$by virtue of the concavity of EOA and the result, $C[(\mathbf{1} \otimes$ $\mathcal{E}) \rho] \leq C(\rho) C\left[(\mathbf{1} \otimes \mathcal{E})\left|\phi^{+}\right\rangle\left\langle\phi^{+}\right|\right]$with $\rho$ a 2-qubit state [8].

This inequality (11) holds for all one-sided channels $\mathcal{E}$. However, assisted entanglement to prepare the entanglement between distant parties sometimes needs to consider the case of two one-sided channels. In fact, the theorem 1 has an immediate generalization for two onesided channels.
Corollary 3 For a two one-sided channel $\mathcal{E}_{1} \times \mathcal{E}_{2}$ acting on Alice and Bob subsystem of a $2 \times 2 \times n_{3}$ state, the evolution equation of EOA leads to an inequality, which provides an upper bound,

$$
\begin{aligned}
& E_{a}\left(\left(\mathcal{E}_{1} \times \mathcal{E}_{2} \times \mathbf{1}\right) \rho_{0}\right) \\
\leq & E_{a}\left(\rho_{0}\right) C\left[\left(\mathbf{1} \otimes \mathcal{E}_{2}\right)\left|\phi^{+}\right\rangle\left\langle\phi^{+}\right|\right] C\left[\left(\mathcal{E}_{1} \otimes \mathbf{1}\right)\left|\phi^{+}\right\rangle\left\langle\phi^{+}\right|\right](12)
\end{aligned}
$$

Due to $\mathcal{E}_{1} \times \mathcal{E}_{2} \times \mathbf{1}=\left(\mathbf{1} \otimes \mathcal{E}_{2} \times \mathbf{1}\right)\left(\mathcal{E}_{1} \times \mathbf{1} \times \mathbf{1}\right)$, we can prove this corollary easily by virtue of the corollary 2 .

Although (11) and (12) are not equalities, the evolution equation of EOA (3) and these upper bounds (11112) are only concerned with the quantity, the entanglement of the final state evolved from a 2-qubit maximally entangled state with the channel's action on it. Hence, these results will ease the experimental characterization of entanglement dynamics of EOA under unknown channels in a preparation of 2-qubit state by assisted entanglement. We, instead of exploring the time-dependent action of the channel on all initial states, only need to investigate the entanglement evolution of the maximally entangled state alone.

The assisted entanglement can also produce a general bipartite entangled state besides 2-qubit states. Moreover, bipartite states with higher dimension can improve the performance of various quantum information and computation tasks. Hence, it is necessary to explore the dynamics of EOA for the characterization of the produced entanglement produced by assisted entanglement when the state is coupled to its environment. In the following section, we explore how EOA evolves for a $d \times d \times n_{3}$ state with local noisy channels operation on it.

\section{EVOLUTION EQUATION OF EOA FOR $d \times d \times n_{3}$ SYSTEMS}

First, we need to define EOA for a $d \times d \times n_{3}$ state. For the consistency with the definition of EOA, we define entanglement of assistance in terms of the entanglement measure I-concurrence [17, 18]:

$$
\begin{aligned}
E_{a}\left(|\psi\rangle_{A B C}\right) & \equiv \max \sum_{i} p_{i} C\left(\left|\phi_{i}\right\rangle_{A B}\right) \\
& =\max \sum_{i} p_{i} \sqrt{\sum_{m n}\left|\left\langle\phi_{i}^{*}\left|S_{m n}\right| \phi_{i}\right\rangle\right|^{2}},
\end{aligned}
$$

which is maximized over all possible pure-state decompositions of $\rho_{A B}=\operatorname{Tr}_{C}\left[|\psi\rangle_{A B C}\langle\psi|\right]=\sum_{i} p_{i}\left|\phi_{i}\right\rangle_{A B}\left\langle\phi_{i}\right|$. where $S_{m n}=L_{m} \otimes L_{n}, L_{m}, m=1, \ldots, d(d-1) / 2$, $L_{n}, n=1, \ldots, d(d-1) / 2$ are the generators of group $S O(d)$. In the same way, EOA, for a mixed tripartite state $\rho_{A B C}$, is defined as the maximum achievable average I-concurrence shared by Alice and Bob after Charlie has performed a local operation and communicated the result to Alice and Bob.

For a general $\rho_{A B C}$ with Bob's system undergoing the action of a noisy channel $\mathcal{E}$, the final state takes the form 
$\rho_{A B C}^{\prime}=(\mathbf{1} \otimes \mathcal{E} \otimes \mathbf{1}) \rho_{A B C}$. Correspondingly, the evolution equation of EOA is obtained as the following theorem.

Theorem 4 The EOA of the final state $\rho_{A B C}^{\prime}$ satisfies the equation,

$$
\begin{aligned}
& E_{a}\left[(\mathbf{1} \otimes \mathcal{E} \otimes \mathbf{1}) \rho_{A B C}\right] \\
\leq & \frac{d}{2} E_{a}\left(\rho_{A B C}\right) C\left[(\mathbf{1} \otimes \mathcal{E})\left|\chi^{+}\right\rangle\left\langle\chi^{+}\right|\right]
\end{aligned}
$$

where $\left|\chi^{+}\right\rangle$is a $d \times d$ maximally entangled state, $\left|\chi^{+}\right\rangle=$ $\sum_{i=0}^{d-1}|i\rangle \otimes|i\rangle / \sqrt{d}$.

Proof: In order to prove (14), we need the result in Ref. 10]. For a $d \times d$ bipartite state $\rho$ with its second subsystem going through a noisy channel $\mathcal{E}$, we have $C[(\mathbf{1} \otimes \mathcal{E}) \rho] \leq \frac{d}{2} C(\rho) C\left[(\mathbf{1} \otimes \mathcal{E})\left|\chi^{+}\right\rangle\left\langle\chi^{+}\right|\right]$.

We first assume that there is a set of optimal measurement $\left\{M_{i}\right\}$ for Charlie such that

$$
E_{a}\left[(\mathbf{1} \otimes \mathcal{E} \otimes \mathbf{1}) \rho_{A B C}\right]=\sum_{i} p_{i} C\left(\rho_{i}\right),
$$

where $p_{i}=\operatorname{Tr}\left[\sum_{j}\left(\mathbf{1} \otimes K_{j} \otimes M_{i}\right) \rho_{A B C}\left(\mathbf{1} \otimes K_{j}^{\dagger} \otimes M_{i}^{\dagger}\right)\right]$, and

$$
\begin{aligned}
\rho_{i} & =\frac{\operatorname{Tr}_{C} \sum_{j}\left(\mathbf{1} \otimes K_{j} \otimes M_{i}\right) \rho_{A B C}\left(\mathbf{1} \otimes K_{j}^{\dagger} \otimes M_{i}^{\dagger}\right)}{p_{i}} \\
& =\sum_{j}\left(\mathbf{1} \otimes K_{j}\right) \tau_{i}\left(\mathbf{1} \otimes K_{j}^{\dagger}\right) .
\end{aligned}
$$

Here $\tau_{i}=\operatorname{Tr}_{C}\left(\left(\mathbf{1} \otimes \mathbf{1} \otimes M_{i}\right) \rho_{A B C}\left(\mathbf{1} \otimes \mathbf{1} \otimes M_{i}^{\dagger}\right)\right) / p_{i}$. Therefore, we have

$$
\begin{aligned}
& E_{a}\left[(\mathbf{1} \otimes \mathcal{E} \otimes \mathbf{1}) \rho_{A B C}\right] \\
= & \sum_{i} p_{i} C\left((\mathbf{1} \otimes \mathcal{E}) \tau_{i}\right) \\
\leq & \frac{d}{2} C\left[(\mathbf{1} \otimes \mathcal{E})\left|\chi^{+}\right\rangle\left\langle\chi^{+}\right|\right] \sum_{i} p_{i} C\left(\tau_{i}\right) \\
\leq & \frac{d}{2} C\left[(\mathbf{1} \otimes \mathcal{E})\left|\chi^{+}\right\rangle\left\langle\chi^{+}\right|\right] E_{a}\left(\rho_{A B C}\right) .
\end{aligned}
$$

Here the last inequality is obtained by the concavity of EOA. From the above analysis, we have proved the theorem.

Remark. In particular, if the initial state is a $d \times 2 \times n_{3}$ pure state, we will obtain an evolution equation of EOA for such a state, $E_{a}\left((\mathbf{1} \otimes \mathcal{E} \otimes \mathbf{1})|\phi\rangle_{A B C}\langle\phi|\right)=$ $E_{a}\left(|\phi\rangle_{A B C}\right) C\left\lceil(\mathbf{1} \otimes \mathcal{E})\left|\phi^{+}\right\rangle\left\langle\phi^{+}\right|\right]$. By the aid of the following equation [10], $C\left[(\mathbf{1} \otimes \mathcal{E})|\psi\rangle_{A B}\langle\psi|\right]=C\left(|\psi\rangle_{A B}\right) C[(\mathbf{1} \otimes$ $\left.\mathcal{E})\left|\phi^{+}\right\rangle\left\langle\phi^{+}\right|\right]$with $|\psi\rangle_{A B}$ a pure $n_{1} \times 2$ state, the evolution equation is then proved in a way similar to that of Theorem 1. If we consider a one-sided channel $\mathcal{E}$ which operates on the third subsystem, we get an lower bound for the EOA of the final state, $E_{a}\left[(\mathbf{1} \otimes \mathbf{1} \otimes \mathcal{E}) \rho_{A B C}\right] \geq$ $C\left(\rho_{A B}\right)$, where $\rho_{A B}=\operatorname{Tr}_{C} \rho_{A B C}$. We can also generalize the Theorem 2 to the case of two one-sided channel $\mathcal{E}_{1} \otimes \mathcal{E}_{2}$, whose result is similar to (14).
Similarly, the dynamics of EOA for a general $d \times d \times n_{3}$ state, which goes through a local one-sided noisy channel, is determined by the channel's action on the bipartite maximally entangled state $\left|\chi^{+}\right\rangle=\sum_{i=0}^{d-1}|i\rangle \otimes|i\rangle / \sqrt{d}$. Therefore, it allows one to understand, without resorting to the channel's action on all initial states, the dynamics of EOA under some local quantum channels for an arbitrary initial state, if one knows the time evolution of entanglement for the bipartite maximally entangled state under such quantum channels.

\section{EXAMPLES AND DISCUSSION}

Let us consider several examples. Suppose $\mathcal{E}$ is a phase damping channel, such that $\mathcal{E}(\rho)=M_{0} \rho M_{0}^{\dagger}+$ $M_{1} \rho M_{1}^{\dagger}$ with $M_{0}=\left(\begin{array}{ll}1 & 0 \\ 0 & \nu\end{array}\right), M_{1}=\left(\begin{array}{cc}0 & 0 \\ 0 & \omega\end{array}\right)$, where the time-dependent parameters $\nu=\exp [-\Gamma t]$ and $\omega=$ $\sqrt{1-\nu^{2}}$. For a generalized GHZ state $\left|\psi_{g}\right\rangle_{A B C}=\alpha|000\rangle+$ $\sqrt{1-|\alpha|^{2}}|111\rangle$, we can get $E_{a}\left[(\mathbf{1} \otimes \mathcal{E} \otimes \mathbf{1})\left|\psi_{g}\right\rangle_{A B C}\left\langle\psi_{g}\right|\right]=$ $C\left[(\mathbf{1} \otimes \mathcal{E})\left|\phi^{+}\right\rangle\left\langle\phi^{+}\right|\right] E_{a}\left(\left|\psi_{g}\right\rangle_{A B C}\right)=2 \exp [-\Gamma t] \alpha \sqrt{1-\left|\alpha^{2}\right|}$ in terms of Eq. (3). Next we suppose the quantum channel is a generalized amplitude damping channel, $\mathcal{E}_{G A D}$, describing the effect of dissipation to an environment at finite temperature. The channel usually adopts the form as: $K_{0}=\sqrt{p}\left(\begin{array}{cc}1 & 0 \\ 0 & \nu\end{array}\right), K_{1}=$ $\sqrt{p}\left(\begin{array}{cc}0 & \omega \\ 0 & 0\end{array}\right), K_{2}=\sqrt{1-p}\left(\begin{array}{cc}\nu & 0 \\ 0 & 1\end{array}\right), K_{3}=\sqrt{1-p}\left(\begin{array}{cc}0 & 0 \\ \omega & 0\end{array}\right)$. Without loss of generality, we set $p=\frac{1}{2}$, then obtain $E_{a}\left[\left(\mathbf{1} \otimes \mathcal{E}_{G A D} \otimes \mathbf{1}\right)\left|\psi_{g}\right\rangle_{A B C}\left\langle\psi_{g}\right|\right]=C^{2}\left(\left(\mathcal{E}_{G A D} \otimes\right.\right.$ 1) $\left.\left|\phi^{+}\right\rangle\left\langle\phi^{+}\right|\right) E_{a}\left(\left|\psi_{g}\right\rangle_{A B C}\right)=|\alpha| \sqrt{1-|\alpha|^{2}}(\exp [-2 \Gamma t]+$ $2 \exp [-\Gamma t]-1)$. From the above equation, we can find there is a sudden death of EOA for this damping channel, similar to the sudden death of entanglement [19 21] . Just like the sudden death of entanglement cannot appear for any channel [19], the sudden death of EOA does not exist for some channels. As shown in Fig. 1, the dashed line, the evolution of $E_{a}\left[(\mathbf{1} \otimes \mathcal{E} \otimes \mathbf{1})\left|\psi_{g}\right\rangle_{A B C}\left\langle\psi_{g}\right|\right]$ with $\mathcal{E}$ a phase damping channel, indicates $E_{a}$ does not die sud-

FIG. 1: The decay of $E_{a}\left[(\mathbf{1} \otimes \mathcal{E} \otimes \mathbf{1})\left|\psi_{g}\right\rangle_{A B C}\left\langle\psi_{g}\right|\right]$ vs $\Gamma t$, where $\Gamma$ is a generalized amplitude decay rate. The dashed line and solid line are the decay of EOA for phase damping channel and generalized amplitude damping channel respectively with $\alpha=\frac{1}{2}$. Note that in solid line a sudden death of EOA appears but in dashed line it does not. 
denly but asymptotically. However, for the generalized amplitude damping channel, the EOA does go abruptly to zero in a finite time and remain zero thereafter. From the above analysis, we can find, under the local quantum channel's action, whether the sudden death of EOA appears depends only on the channel's action on maximally entangled states.

The inequality $C_{a}^{2}\left(\rho_{A B}\right)+C_{a}^{2}\left(\rho_{A C}\right)-C^{2}\left(|\psi\rangle_{A-B C}\right)=$ $C^{2}\left(|\psi\rangle_{A-B C}\right)-C^{2}\left(\rho_{A B}\right)-C^{2}\left(\rho_{A C}\right) \geq 0$ holds [12]. First, we denote the quantity by $\tau\left(\rho_{A B C}\right), \tau\left(\rho_{A B C}\right)=$ $C_{a}^{2}(\rho(A B))+C_{a}^{2}(\rho(A C))-C^{2}\left(\rho_{A-B C}\right)$, which is just the three-tangle [22] if $\rho_{A B C}$ is pure. We consider a case that a mixed state $\rho$ can be rewritten as $\rho=$ $\left.\sum_{(} K_{i} \otimes \mathbf{1} \otimes \mathbf{1}\right)|\psi\rangle_{A B C}\langle\psi|\left(K_{i}^{\dagger} \otimes \mathbf{1} \otimes \mathbf{1}\right)$ with $\left\{K_{i}\right\}$ representing the operation of a quantum channel $\mathcal{E}$, i.e. an output state evolved from the initial state $|\psi\rangle_{A B C}$ of which the first subsystem goes through a quantum channel $\mathcal{E}$. Then, according to the Theorem 1 and the result in Ref. [10], we obtain the following equations, $\tau\left((\mathcal{E} \otimes \mathbf{1} \otimes \mathbf{1})|\psi\rangle_{A B C}\langle\psi|\right)=C_{a}^{2}(\rho(A B))+C_{a}^{2}(\rho(A C))-$ $C^{2}\left(\rho_{A-B C}\right)=C^{2}\left((\mathcal{E} \otimes \mathbf{1})\left|\phi^{+}\right\rangle\left\langle\phi^{+}\right|\right)\left[C_{a}^{2}\left(\rho_{A B}\right)+C_{a}^{2}\left(\rho_{A C}\right)-\right.$ $\left.C^{2}\left(|\psi\rangle_{A-B C}\right)\right]=C^{2}\left((\mathcal{E} \otimes \mathbf{1})\left|\phi^{+}\right\rangle\left\langle\phi^{+}\right|\right) \tau\left(|\psi\rangle_{A B C}\right) \geq 0$. Up to now, however, no references provide a proof whether or not the inequality $C_{a}^{2}(\rho(A B))+C_{a}^{2}(\rho(A C))-$ $C^{2}\left(\rho_{A-B C}\right) \geq 0$ is always satisfied for a general mixed 3 -qubit state $\rho_{A B C}$. Although we only prove the quantity $\tau$ is greater than or equal to zero for such a mixed 3-qubit state, we are willing to conjecture that it is still valid for any mixed 3-qubit states.

\section{SUMMARY}

In summary, we have investigated the time evolution of EOA when one subsystem undergoes the action of an ar- bitrary noisy channel. In particular, for a $2 \times 2 \times n_{3}$ pure quantum state with the qubit subsystem being subject to a noisy channel, we get a general factorization law for evolution equation of EOA. Furthermore, for the other cases with one subsystem undergoing the action of local quantum channels, a similar relation is satisfied, at a price of turning the factorization law into an inequality. We use generalized amplitude damping and phase damping channels as examples and find that the sudden death of EOA does exist in the evolution and is only determined by the entanglement evolution equation of the maximally entangled state entering this channel. As a result, in order to characterize the dynamics of EOA, we, instead of exploring the time-dependent action of the channel on all initial states, only need to investigate the entanglement evolution of the maximally entangled state, which is universal for all initial states passing through this channel. Hence, these results will ease the experimental characterization of entanglement dynamics of EOA under unknown channels in an experimental preparation of a bipartite state by assisted entanglement.
[1] R. Horodecki, P. Horodecki, and M. Horodecki, Rev. Mod. Phys. 81, 865 (2009).

[2] M. A. Nielsen and I. L. Chuang, Quantum Computation and Quantum Information (Cambridge University Press, Cambridge, 2000).

[3] F. Verstraete, M. Popp, and J. I. Cirac, Phys. Rev. Lett. 92, 027901 (2004); M. Popp, F. Verstraete, M. A. MartinDelgado, and J. I. Cirac, Phys. Rev. A 71, 042306 (2005).

[4] H. J. Briegel, W. Dür, J. I. Cirac, and P. Zoller, Phys. Rev. Lett. 81, 5932 (1998).

[5] D. P. DiVincenzo, C. A. Fuchs, H. Mabuchi, J. A. Smolin, A. Thapliyal, and A. Uhlmann, The Entanglement of Assistance, Lecture Notes in Computer Science Vol. 1509 (Springer-Verlag, Berlin, 1999), pp. 247-257

[6] O. Cohen, Phys. Rev. Lett. 80, 2493 (1998).

[7] D. M. Greenberger, M. Horne, and A. Zeilinger, Bell's Theorem, Quantum Theory, and Conceptions of the Universe, edited by M. Kafatos (Kluwer, Dordrecht, 1989) p. 69.

[8] T. Konrad, F. De Melo, M. Tiersch, C. Kasztelan, A. Aragão, and A. Buchleitner, Nature Phys. 4, 99 (2008).
[9] M. Tiersch, F. De Melo, and A. Buchleitner, Phys. Rev. Lett. 101, 170502 (2008).

[10] Z. G. Li, S. M. Fei, Z. D. Wang and W. M. Liu, Phys. Rev. A 79, 024303 (2009).

[11] T. Laustsen, F. Verstraete, and S. J. van Enk, Quantum Inf. Comput. 3, 64 (2003).

[12] G. Gour, D. A. Meyer, and B. C. Sanders, Phys. Rev. A 72, 042329 (2005).

[13] W. K. Wootters, Phys. Rev. Lett. 80, 2245 (1998).

[14] L. P. Hughston, R. Jozsa, and W. K. Wootters, Phys. Lett. A 183, 14 (1993).

[15] G. Gour, Phys. Rev. A 72, 042318 (2005).

[16] Thomas Konrad et al. [8] have proved that, for a twoqubit pure state $|\psi\rangle$, after only one qubit goes through an arbitrary channel $\mathcal{E}$, the concurrence decays just by a universal factor only determined by $\mathcal{E}_{\mathrm{S}}$ action on the maximally entangled state, $C((\mathbf{1} \otimes \mathcal{E})|\psi\rangle)=C(|\psi\rangle) C((\mathbf{1} \otimes$ $\left.\mathcal{E})\left|\phi^{+}\right\rangle\right)$. For an arbitrary two-qubit pure state $|\psi\rangle$, the following equation is always satisfied, $|\psi\rangle=\left(M_{\psi} \otimes\right.$ 1) $\left|\phi^{+}\right\rangle\left\langle\phi^{+}\right|\left(M_{\psi}^{\dagger} \otimes \mathbf{1}\right)$, with the elements of matrix $M_{\psi}$ 
the coefficients of the pure state $|\psi\rangle$. Therefore, one can obtain $C((\mathbf{1} \otimes \mathcal{E})|\psi\rangle)=C\left(\left(M_{\psi} \otimes \mathbf{1}\right) \rho_{\mathcal{E}}\left(M_{\psi}^{\dagger} \otimes \mathbf{1}\right)\right)=$ $C(|\psi\rangle) C\left(\rho_{\mathcal{E}}\right)$, where $\rho_{\mathcal{E}}=(\mathbf{1} \otimes \mathcal{E})\left|\phi^{+}\right\rangle\left\langle\phi^{+}\right|$.

[17] P. Rungta, V. Bužek, C. M. Caves, M. Hillery, and G. J. Milburn, Phys. Rev. A 64, 042315 (2001).

[18] Z. G. Li, S. M. Fei, S. Albeverio and W. M. Liu, Phys. Rev. A 80, 034301 (2009).

[19] T. Yu and J. H. Eberly, Phys. Rev. Lett. 97, 140403
(2006)

[20] M. P. Almeida, F. de Melo, M. Hor-Meyll, A. Salles, S. P. Walborn, P. H. Souto Ribeiro, and L. Davidovich, Science 316, 579 (2007).

[21] T. Yu and J. H. Eberly, Science 323, 598 (2009).

[22] V. Coffman, J. Kundu, and W. K. Wootters, Phys. Rev. A 61, 052306 (2000). 